\title{
Can a stationary Bianchi black brane have momentum along the direction with no translational symmetry?
}

\author{
Norihiro lizuka, ${ }^{a}$ Akihiro Ishibashi ${ }^{b}$ and Kengo Maeda ${ }^{c}$ \\ ${ }^{a}$ Interdisciplinary Fundamental Physics Team, Interdisciplinary, \\ Theoretical Science Research Group, RIKEN, Wako 351-0198, Japan \\ ${ }^{b}$ Department of Physics, Kinki University, \\ Higashi-Osaka 577-8502, Japan \\ ${ }^{c}$ Faculty of Engineering, Shibaura Institute of Technology, \\ Saitama 330-8570, Japan \\ E-mail: norihiro.iizuka@riken.jp, akihiro@phys.kindai.ac.jp, \\ maeda302@sic.shibaura-it.ac.jp
}

ABSTRACT: Bianchi black branes (black brane solutions with homogeneous but anisotropic horizons classified by the Bianchi type) provide a simple holographic setting with lattice structures taken into account. In the case of holographic superconductor, we have a persistent current with lattices. Accordingly, we expect that in the dual gravity side, a black brane should carry some momentum along a direction of lattice structure, where translational invariance is broken. Motivated by this expectation, we consider whether - and if possible, in what circumstances - a Bianchi black brane can have momentum along a direction of no-translational invariance. First, we show that this cannot be the case for a certain class of stationary Bianchi black brane solutions in the Einstein-Maxwell-dilation theory. Then we also show that this can be the case for some Bianchi $\mathrm{VII}_{0}$ black branes by numerically constructing such a solution in the Einstein-Maxwell theory with an additional vector field having a source term. The horizon of this solution admits a translational invariance on the horizon and conveys momentum (and is "rotating" when compactified). However this translational invariance is broken just outside the horizon. This indicates the existence of a black brane solution which is regular but non-analytic at the horizon, thereby evading the black hole rigidity theorem.

KEYwORDS: Gauge-gravity correspondence, AdS-CFT Correspondence, Holography and condensed matter physics (AdS/CMT)

ARXIV EPRINT: 1403.0752

\footnotetext{
${ }^{1}$ Address after April 1, 2014: Department of Physics, Osaka University, 560-0043, Japan.
} 


\section{Contents}

1 Introduction $\quad 1$

2 Symmetries on the horizon $\quad 4$

3 Symmetries in the bulk: a no-go in Einstein-Maxwell-dilaton theory 6

$\begin{array}{ll}3.1 \text { Bianchi } \mathrm{VII}_{0} \text { model } & 7\end{array}$

$\begin{array}{llr}3.2 & \text { Bianchi } \mathrm{VI}_{0} & 10\end{array}$

$\begin{array}{lll}3.3 & \text { Bianchi II } & 10\end{array}$

4 Symmetries broken in the bulk, restored on the horizon 11

$\begin{array}{lll}4.1 \text { Our model } & 11\end{array}$

$\begin{array}{ll}4.2 \text { Equations of motion } & 12\end{array}$

$\begin{array}{lll}4.3 & \text { Numerical solution } & 14\end{array}$

$\begin{array}{llr}5 & \text { Summary and discussion } & 17\end{array}$

$\begin{array}{lr}\text { A Bianchi classification } & 18\end{array}$

\section{Introduction}

Recent developments of revealing a rich class of new IR structure of black holes/branes show that various different classes of field theory dynamics could be realized in black hole/brane solutions in gravity side. One of these examples is holographic superconductor/superfluidity [1-6]. Symmetry breaking in the IR in gauge theory is reflected as various new hairy black brane solutions in gravity. Another interesting black brane solutions are Lifshitz geometry [7-10] and so-called geometry with hyperscaling violation [11-22]. There, geometry shows more anisotropic solution in the sense that scaling dimension is more generic than Lorentz-invariant case, but respects spatial rotation and translational invariance in the IR. Therefore these can be bulk dual to homogeneous and isotropic but not necessary Lorentz invariant field theory. Furthermore, these geometries can admit vanishing entropy density at the zero temperature limit, which is natural from thermodynamical point of view. As an attempt to explore various IR geometries, the 5-dimensional extremal black brane geometries which admit homogeneous but anisotropic in IR are classified using the so-called 3-dimensional "Bianchi"-classification [23]. More generic exotic brane classification, using 4-dimensional extension of 3-dimensional Bianchi-classification, was done in 3-dimensional Bianchi-classification [24]. These correspond to the vacuum classification in the boundary gauge theories.

These Bianchi black branes are interesting since they do not in general admit translational invariance, but rather admit non-commutative three Killing vectors $\xi_{i}$ that obey 
nontrivial Lie algebra. Since $\partial_{x^{i}}$, a spatial shift translation along $x^{i}$, does not necessarily coincide a Killing vector except Bianchi I, the geometry does not in general have a translational invariance along the direction of $x^{i}$. An especially interesting class is of type $\mathrm{VII}_{0}$, in which the three-dimensional homogeneous space forms a periodic "helical structure". As pointed out by [25], the directions with no translational invariance can be regarded as "latticed directions". Lattice effects which break the translational invariance are especially interesting and important in realistic condensed matter systems because momentum dissipation occurs due to the lattice. Quite recently, we have shown that there exists a new stationary hairy Bianchi $\mathrm{VII}_{0}$ black brane solution dual to a superconducting state in the strongly coupled field theory side and the angular momentum exists along the "latticed direction" [26] (See also the recent analysis of lattice effects in holographic superconductors $[27,28])$. This implies that persistent current exists along the latticed direction and its resistance becomes zero even though there is no translational invariance along the direction of the current.

From general relativistic point of view, it is also interesting to consider in what circumstances a black brane can have momentum (if possible) along a direction with no translational symmetry. In the asymptotically flat case, one can speculate what would happen when an asymmetric black hole rotates: it would emit gravitational waves that carry angular momentum away, and eventually the rotation of the black hole would be damped out and the geometry would approach a static solution. In other words, as long as it is rotating, an asymmetric black hole will never be exactly stationary. This view is closely related to a consequence of the black hole rigidity theorem [29-31] that a stationary rotating black hole must be axisymmetric. However, in the asymptotically AdS black hole case, which is more relevant to the holographic context, the emitted gravitational radiation would be reflected back by the AdS infinity and its backreaction could possibly make the geometry either a state of forever dynamical (see, e.g., [32-35]) or an equilibrium state with no axisymmetry due to the presence of gravitational waves outside the horizon, besides the possibility that the geometry would settle down to another stationary, axisymmetric solution. In particular, for the equilibrium, non-axisymmetric case, the event horizon itself does not rotate with respect to the generator of the stationary symmetry according to the rigidity theorem, but instead some radiation outside the horizon would carry the angular momentum, presumably making the bulk geometry non-static. If some matter fields are included, they could also be a carrier of (part of) the angular momentum. (This is in fact the case in our previous paper [26]. See also an earlier result along this line [36]). Furthermore, in the context of gauge/gravity duality, we are typically concerned with black branes with non-compact horizon, to which the standard form of the rigidity theorem no longer applies, as the compactness of horizon cross-sections is one of the essential requirements for the proof of the theorem. (See also references $[37,38]$ for constructions of black branes that evade the rigidity theorem by considering non-compact horizons.) It is therefore worth considering what would happen when a black brane, whose horizon cross-sections are not necessarily compact, gains momentum along a direction of no translational symmetry. For this line of study, the Bianchi black brane models described above provide a good starting 
point since, as we will see later, the equations of motion for the Bianchi models reduce to a set of ordinary differential equations.

The purpose of this paper is to reveal some non-trivial relation between the symmetry and momentum of a certain class of Bianchi black brane models that are expected to be dual to strongly coupled quantum systems, such as superconductors with lattices. We will supply a new example of Bianchi black brane solutions in the Einstein-Maxwell theory with an additional vector field. In our previous paper [26], we have constructed a stationary hairy Bianchi $\mathrm{VII}_{0}$ black brane solution, in which the horizon is not rotating but matter fields carry momentum. The point of our construction in [26] is that a $U(1)$ gauge field acquires a mass, which is a desired phenomenon in accord with a superconducting state in the dual theory side. Then one might expect that a Bianchi black brane cannot carry momentum unless a gauge field of the model becomes massive. We will show that this is indeed the case in the same type of Bianchi black branes considered in [26] in EinsteinMaxwell and Einstein-Maxwell-dilaton theories.

We shall also explore another possibility that a black brane can have momentum along a direction of no translational invariance even in the case that a gauge field involved does not gain a mass. We will numerically construct such an example for black branes of Bianchi $\mathrm{VII}_{0}$ type, by considering a model with a source term in the bulk Lagrangian. In this solution, the horizon can be compactified and is in fact "rotating" (when compactified) along the direction of translational invariance on the horizon and hence conveys momentum. However, this translational invariance is actually violated just outside the horizon. This would be impossible if the metric and fields are analytic in a neighbourhood of the horizon, since in that case, the geometry - in particular, its symmetry property - near the horizon can be uniquely extended into the entire region by analytic continuation (under an additional condition of simple connectedness). Therefore this solution indicates, within the accuracy of our numerical method, the existence of a black brane which is regular but is not analytic at the horizon. The analyticity is a crucial requirement for the proof of rigidity theorem, besides the compactness of the horizon cross-sections. Therefore, although a mathematically more rigorous proof for this to be truly so is desired, our solution provides a qualitatively new, intriguing example of regular black branes which possess a stationary Killing vector field tangent to the horizon but nevertheless evade the rigidity theorem by the violation of analyticity.

The organization of this paper is as follows. In the next section, we show a theorem concerning the decomposition of Killing symmetry on the event horizon in a class of stationary Bianchi black branes. In section 3, we show that Einstein-Maxwell theory or Einstein-Maxwell-dilaton theory cannot have a rotating black brane solution with momentum/rotation along the direction of no translational invariance for the same class of stationary Bianchi black branes considered in section 2. This corresponds to the lack of persistent current in normal phase (non-superconductor) along the direction of no translational invariance. In section 4 , we illustrate a model with a source term in the bulk Lagrangian which generates an explicit stationary solution where the horizon is rotating along the direction of no translational invariance except the horizon. Section 5 is devoted to summary and discussion. 


\section{Symmetries on the horizon}

In this section and the next, we show that the event horizon in a certain class of stationary 5-dimensional Bianchi black branes cannot rotate along the direction of no translational invariance. As discussed above, this is reminiscent of the black hole rigidity theorem. However it should be noted that the rigidity theorem requires, in an essential way, the compactness of the horizon cross-sections. In contrast, we will here deal with stationary black brane whose horizon cross-sections are not necessarily compact. In this section we discuss symmetry aspects focusing on the horizon, without any restriction to the type of theories, other than the requirement of null convergence condition. In the next section we turn our attention to the exterior of the horizon and consider momentum carried by matter fields within Einstein-Maxwell(-dilaton) theory.

In what follows, by 5 -dimensional stationary Bianchi black brane we mean a stationary geometry containing a black hole, whose exterior region can be foliated by 4-dimensional timelike homogeneous hypersurfaces $\mathcal{N}(r)$, labeled by a parameter $r$ and spanned by mutually independent four Killing vector fields, $k$ and $\xi^{I}(I=1,2,3)$. Here $k$ is an asymptotically timelike Killing vector field with complete orbits, and $\xi^{I}$ are assumed to be everywhere spacelike, commute with $k$, and satisfy the Lie algebra

$$
\left[\xi_{I}, \xi_{J}\right]=C^{K}{ }_{I J} \xi_{K}
$$

in the Bianchi classification (see appendix A in detail). Then, with the foliation parameter $r$ suitably chosen as the radial coordinate and the Killing parameter $t$ of $k=\partial_{t}$ as the time coordinate, the 5 -dimensional metric is written as

$$
d s_{5}^{2}=-h d t^{2}+\frac{d r^{2}}{f}+2 \tilde{N}_{I} d t \omega^{I}+\tilde{g}_{I J} \omega^{I} \omega^{J}, \quad I, J=1,2,3,
$$

where $h, f, \tilde{N}_{I}$, and $\tilde{g}_{I J}$ are functions of $r$ only, and each $\omega^{I}$ denotes the invariant oneform associated with $\xi_{I}$. When the shift vector $\tilde{N}_{I}$ is non-vanishing, the spacetime can possess momentum/rotate with respect to the asymptotic observers along the orbits of $k$. When the horizon is rotating, $h$ is negative since $k$ becomes spacelike on the horizon, i.e., $g_{5}(k, k)>0$. Let us denote the location of the horizon $H$ by $r=r_{h}\left(f\left(r_{h}\right)=0\right)$. Then, the horizon $H$ consists of a homogeneous null hypersurface spanned by $\xi^{I}$ and $k$ in the limit, $r \rightarrow r_{h}$, of $\mathcal{N}$. Now choose a horizon cross-section $\Sigma$ of $H$ in a way that on $H$, $k$ is everywhere transverse to $\Sigma$. Then Lie-dragging $\Sigma$ by $k$ defines a foliation $\Sigma(u)$ of $H$, where the parameter $u$ is given so that $\mathcal{L}_{k} u=1$.

Theorem 1. Consider a 5-dimensional stationary black brane with the stationary Killing vector $k$, and choose a horizon cross-section $\Sigma$ in the manner described above. Then $k$ is uniquely decomposed into a null vector field $n$ normal to $H$ and a spacelike vector $\eta$ lies in $\Sigma$ as

$$
k=n-\eta .
$$

Now suppose $\Sigma$ admits the Bianchi symmetry of the type II, VII , or VII $I_{0}$. Then, under the null energy condition, either $\eta=0$, or $\eta$ must be a Killing vector of $\Sigma$, irrespective of $\Sigma$ being compact or non-compact. 
Proof. Since $k$ generates an isometry, it must be tangent to $H$. When $k$ is normal to $H, \eta$ is identically zero. So, hereafter we focus on the case that $k$ is spacelike on $H$ and hence $N^{2}:=g_{5}(k, k)>0$ on $H$. Since the Killing vectors $k$ and $\xi^{I}$ all tangent to $H$, the metric of $H$ may be written by a local coordinate $u$ with $\mathcal{L}_{k} u=1$ as

$$
d s_{4}^{2}=N^{2} d u^{2}+2 N N_{I} \omega^{I} d u+g_{I J} \omega^{I} \omega^{J},
$$

where $N, N_{I}$, and $g_{I J}$ are constants satisfying $\operatorname{det} g_{I J}>0$ (in addition to $N^{2}>0$ mentioned above) and where, furthermore, the determinant of the gram matrix of $k$ and $\xi_{I}$ is required to vanish. If one expands $\eta$ by the invariant vectors $X_{I}$ as $\eta=\alpha^{I} X_{I}$, then the coefficients $\alpha^{I}\left(=g^{I J} \alpha_{J}\right)$ is uniquely determined by

$$
\alpha_{I}=-N N_{I}, \quad N_{I} N^{I}=1
$$

by the conditions $g_{4}(n, n)=0$ and $\partial_{\alpha_{I}} g_{4}(n, n)=0$, as $H$ is an achronal surface. ${ }^{1}$ It is straightforward to check that the gramian in fact vanishes.

Now let us consider a symmetric tensor $B_{i j}$ defined by $B_{i j}:=\widehat{\nabla}_{i} l_{j}(i, j=1,2,3)$ with $l$ being the tangent vector for the null geodesic generator of $H$ with an affine parameter $\lambda$, where the hat means the projection onto the spatial cross-section $\Sigma,{ }^{2}$ and here and hereafter the indices $i, j=1,2,3$ denote local coordinates $x^{i}$ on $\Sigma$. Then, the expansion $\theta$ and the shear tensor $\sigma_{i j}$ are defined by

$$
\theta=B_{i}{ }^{i}, \quad \sigma_{i j}=B_{i j}-\frac{1}{3} \theta g_{i j}=\frac{1}{2} \mathcal{L}_{l} g_{i j}-\frac{1}{3} \theta g_{i j}
$$

where $g_{i j}$ is the induced metric of $\Sigma$. By rewriting $l$ as $l=f n$ with some appropriate function $f$, the expansion $\theta$ is calculated as

$$
\theta=g^{i j} B_{i j}=\frac{1}{2} f g^{i j} n^{\mu} \partial_{\mu} g_{i j}+f \alpha^{I} \partial_{i} X_{I}^{i}
$$

where we used $g_{4}\left(n, \partial_{i}\right)=0$. Note that if the horizon cross-section is compact, hence the area of $\Sigma$ is well-defined, then one can immediately show that $\theta=0$ under the null convergence condition by exploiting type of arguments used for the area-theorem [29]. However we do not proceed in that way, as we are interested also in the case where the horizon cross-sections are non-compact.

Now here we use some particular property of the Bianchi type II, $\mathrm{VI}_{0}, \mathrm{VII}_{0}$ : for those, we find that the divergence, $\partial_{i} X_{I}{ }^{i}$, of the invariant vector $X_{I}$ vanishes for an arbitrary index $I$. Using this fact, we then find that

$$
\theta=\frac{1}{2} f g^{i j} n^{\mu} \partial_{\mu} g_{i j}=\frac{1}{2 A} \frac{d A}{d \lambda}
$$

where $A=\operatorname{det}\left(g_{i j}\right)$. In terms of the local coordinates $x^{i}, g_{i j}$ on $\Sigma$ is given by

$$
\begin{aligned}
d s_{3}^{2} & =\widehat{g_{I J}} \omega^{I}(x) \omega^{J}(x)=g_{i j}(x) d x^{i} d x^{j}, & I, J & =1,2,3, \\
g_{i j}(x) & =\widehat{g_{I J}} \Lambda^{I}{ }_{i}(x) \Lambda^{J}{ }_{j}(x), & \omega^{I}(x) & :=\Lambda^{I}{ }_{i}(x) d x^{i} .
\end{aligned}
$$

\footnotetext{
${ }^{1}$ An achronal surface is a surface which contains no two points with timelike separation. The only non-spacelike curve that can lie in $H$ is a null geodesic on $H$.

${ }^{2}$ Since $l$ is a hypersurface orthogonal, the anti-symmetric part of $B_{i j}$ automatically vanishes.
} 
Along each null geodesic congruence of $l$, the Raychaudhuri equation holds:

$$
\frac{d \theta}{d \lambda}=-\sigma_{i j} \sigma^{i j}-R_{\mu \nu} l^{\mu} l^{\nu}-\frac{1}{3} \theta^{2} .
$$

Here we use again a particular property of Bianchi II, $\mathrm{VI}_{0}, \mathrm{VII}_{0}$ : for those types, one can find that besides the fact that $\widehat{g_{I J}}$ is a constant matrix on $H$, the $\operatorname{determinant} \operatorname{det} \Lambda^{I}{ }_{i}(x)$ also becomes constant. Then it follows that $A$ also must be constant, and hence $\theta=0$ along $H$. This immediately yields

$$
R_{\mu \nu} l^{\mu} l^{\nu}=-\sigma_{i j} \sigma^{i j}
$$

Since the null energy condition is imposed, it must be that $R_{\mu \nu} l^{\mu} l^{\nu} \geq 0$. This would imply that $2 \sigma_{i j}=\mathcal{L}_{l} g_{i j}=0$ for any $i$ and $j$. By using $l=f n$ and $g_{4}\left(\partial_{i}, n\right)=0$, we have

$$
\mathcal{L}_{l} g_{i j}=f \mathcal{L}_{n} g_{i j}=f \mathcal{L}_{\eta} g_{i j}=0 .
$$

Therefore $\eta$ must be a Killing vector on $\Sigma$.

It is worth mentioning to the other Bianchi models. In general, $\theta \neq 0$, as $\operatorname{det} \Lambda^{I}{ }_{i}$ depends on the local coordinate $x^{i}$. In this case, $\theta$ must be positive in the future direction according to the area theorem or the second law of the thermodynamics, implying that the entropy density continues to increase. As suggested in the inhomogeneous models [37, 38], such a dissipating stationary black brane solution could exist even in the homogeneous case.

\section{Symmetries in the bulk: a no-go in Einstein-Maxwell-dilaton theory}

In this section, we will complete our proof that momentum must be zero along the directions with no translational symmetry by showing that the shift vector $\tilde{N}_{I}$ in eq. (2.2) is identically zero outside the horizon. As we mentioned in section 1, the proof crucially depends on whether gauge field acquires a mass or not. In fact, if $U(1)$ gauge field acquires the mass, the gauge field outside the horizon can possess a momentum along the direction with no translational symmetry [26].

Since we are interested in the case where the mass of the gauge field is zero, we proceed by restricting ourselves to the following Einstein-Maxwell (dilaton) theory:

$$
S=\int d^{5} x \sqrt{-g}\left(R-(\nabla \phi)^{2}-\frac{1}{4} e^{2 \alpha \phi} F^{2}-\frac{1}{4} W^{2}-V(\phi)\right)
$$

where $A_{\mu}$ and $B_{\mu}$ are gauge potential one-forms and their field strengths are $F=d A$ and $W=d B$, respectively. Here, $\alpha$ is an arbitrary parameter and $V(\phi)$ is an arbitrary potential of the dilaton field $\phi$ such that $V$ has an extreme value $-12 / L^{2}$ at $\phi=\phi_{0}$ and satisfies the $\mathrm{BF}$ bound. The one-form $B$ plays a role for inducing the helical lattice effect on the bulk in Bianchi $\mathrm{VII}_{0}$ black brane spacetime, as shown in $[25,26]$. 
The equations of motion are

$$
\begin{gathered}
R_{\mu \nu}=\nabla_{\mu} \phi \nabla_{\nu} \phi+\frac{1}{2} e^{2 \alpha \phi} F_{\mu \rho} F_{\nu}{ }^{\rho}+\frac{1}{2} W_{\mu \rho} W_{\nu}{ }^{\rho} \\
-g_{\mu \nu}\left(\frac{1}{12}\left(e^{2 \alpha \phi} F^{2}+W^{2}\right)-\frac{1}{3} V(\phi)\right), \\
\sqrt{-g} \nabla_{\nu}\left(e^{2 \alpha \phi} F^{\mu \nu}\right)=\partial_{\nu}\left(\sqrt{-g} e^{2 \alpha \phi} F^{\mu \nu}\right)=0, \\
\sqrt{-g} \nabla_{\nu} W^{\mu \nu}=\partial_{\nu}\left(\sqrt{-g} W^{\mu \nu}\right)=0, \\
\nabla^{2} \phi-\frac{1}{4} \alpha e^{2 \alpha \phi} F^{2}-\frac{1}{2} \frac{\partial V}{\partial \phi}=0 .
\end{gathered}
$$

To simplify the analysis, we shall restrict the metric (2.2) into the following particular form

$$
d s^{2}=-f d t^{2}+\frac{d r^{2}}{f}+e^{2 v_{3}}\left(d x^{1}+\Omega d t\right)^{2}+e^{2 v_{1}}\left(\omega^{1}\right)^{2}+e^{2 v_{2}}\left(\omega^{2}\right)^{2}
$$

where $\partial_{x^{1}}$ is a direction of no translational symmetry, as explained below. We assume $f, \Omega, v_{i}$ are functions of $r$ only. In general, the asymptotic behavior of the functions $f$ and $v_{i}$ are given by

$$
f=\frac{r^{2}}{L^{2}}+O(1), \quad v_{i} \simeq \ln r+\frac{1}{2} \ln C_{i}, \quad \Omega=\Omega_{0}+\frac{\Omega_{1}}{r^{4}},
$$

where $\Omega_{0}, \Omega_{1}$ are constants and $C_{i}$ are positive constants. Since we are seeking for a solution where momentum flow occurs without non-normalizable mode, we shall impose

$$
\Omega_{0}=0
$$

as an asymptotic condition for $\Omega$.

Note that the subleading constant term in $f$ comes from the curvature of the three dimensional homogeneous Bianchi space. So, strictly speaking, the geometry does not asymptotically approach AdS spacetime, which is conformally flat. However, in this paper, let us simply call such a geometry by asymptotically "AdS" spacetime in the sense that the leading terms in the metric approach AdS spacetime. In the following subsections, we will show that $\Omega$ is identically zero in Bianchi II, $\mathrm{VI}_{0}$, and $\mathrm{VII}_{0}$.

\subsection{Bianchi $\mathrm{VII}_{0}$ model}

Let us start with most interesting case, Bianchi type $\mathrm{VII}_{0}$. Bianchi type $\mathrm{VII}_{0}$ is defined in (A.7), which has three Killing vectors,

$$
\xi_{1}=\partial_{x^{2}}, \quad \xi_{2}=\partial_{x^{3}}, \quad \xi_{3}=\partial_{x^{1}}-x^{3} \partial_{x^{2}}+x^{2} \partial_{x^{3}},
$$

and three invariant one-forms,

$$
\omega^{1}=\cos \left(x^{1}\right) d x^{2}+\sin \left(x^{1}\right) d x^{3}, \omega^{2}=-\sin \left(x^{1}\right) d x^{2}+\cos \left(x^{1}\right) d x^{3}, \omega^{3}=d x^{1} .
$$


From these, it is clear that along $x^{1}$, there is no translational invariance, $x^{1} \rightarrow x^{1}+$ const.

Following [26], we make the following ansatz for the Maxwell field, the vector field $B$, and the dilaton field $\phi$,

$$
A_{\mu} d x^{\mu}=A_{t}(r) d t+A_{x^{1}}(r) \omega^{3}, \quad B_{\mu} d x^{\mu}=b(r) \omega^{1}, \quad \phi=\phi(r) .
$$

Under the ansatz, we obtain equations of motion for $A_{t}$ and $A_{x^{1}}$ as

$$
\begin{aligned}
\partial_{r}\left(e^{v_{1}+v_{2}+v_{3}+2 \alpha \phi}\left(A_{t}^{\prime}-\Omega A_{x^{1}}^{\prime}\right)\right) & =0, \\
\partial_{r}\left(e^{v_{1}+v_{2}+v_{3}+2 \alpha \phi}\left\{\Omega\left(A_{t}^{\prime}-\Omega A_{x^{1}}^{\prime}\right)+f e^{-2 v_{3}} A_{x^{1}}^{\prime}\right\}\right) & =0,
\end{aligned}
$$

where here and hereafter the prime denotes the derivative with respect to $r$. These equations are integrated to

$$
\begin{aligned}
e^{v_{1}+v_{2}+v_{3}+2 \alpha \phi}\left(A_{t}^{\prime}-\Omega A_{x^{1}}^{\prime}\right) & =\beta_{0}, \\
e^{v_{1}+v_{2}+v_{3}+2 \alpha \phi}\left\{\Omega\left(A_{t}^{\prime}-\Omega A_{x^{1}}^{\prime}\right)+f e^{-2 v_{3}} A_{x^{1}}^{\prime}\right\} & =C,
\end{aligned}
$$

where $\beta_{0}$ and $C$ are integration constants. By the asymptotic conditions (3.7), (3.8), and $\phi \rightarrow \phi_{0}$, we obtain $A_{t}^{\prime}(r \rightarrow \infty) \sim \beta_{0} / r^{3}$. So, $\beta_{0}$ corresponds to the expectation value of the charge density, $\left\langle j_{t}\right\rangle$ in the dual field theory by the AdS/CFT duality. Since we are interested in the dual field theory where charge density or charge current exists, hereafter, we assume that $\beta_{0} \neq 0$.

Eliminating $A_{t}$ from these two equations (3.14), (3.15), one obtains

$$
\Omega \beta_{0}+f e^{2 \alpha \phi} e^{v_{1}+v_{2}-v_{3}} A_{x^{1}}^{\prime}=C .
$$

Below we discuss the two cases, the one $v_{1} \neq v_{2}$ and the other $v_{1}=v_{2}$, on the horizon, separately.

Now, first consider the generic case, $v_{1} \neq v_{2}$ on the horizon. Then, $\partial_{x^{1}}$ is the direction of no translational invariance and hence $\Omega_{h}=0$ by the theorem in section 2 . This implies that $C=0$, as $f=0$ on $H$. The equation of motion for $\Omega$ is obtained from the $\left(t, x^{1}\right)$ and $\left(x^{1}, x^{1}\right)$ component of the Einstein equation (3.2) by eliminating $v_{3}^{\prime \prime}$, as

$$
\begin{aligned}
f \Omega^{\prime \prime} & +f\left(v_{1}^{\prime}+v_{2}^{\prime}+3 v_{3}^{\prime}\right) \Omega^{\prime} \\
& -e^{-2\left(v_{2}+v_{3}\right)}\left[e^{2 v_{2}}\left(4 \Omega \sinh ^{2}\left(v_{1}-v_{2}\right)-e^{2 \alpha \phi} f A_{x^{1}}^{\prime}\left(A_{t}^{\prime}-\Omega A_{x^{1}}^{\prime}\right)\right)+b^{2} \Omega\right]=0 .
\end{aligned}
$$

Eliminating $A_{x^{1}}^{\prime}$ and $A_{t}^{\prime}$ from eqs. (3.14) and (3.16) under the condition $C=0$, we obtain a linear differential equation with respect to $\Omega$,

$$
\Omega^{\prime \prime}+\left(v_{1}^{\prime}+v_{2}^{\prime}+3 v_{3}^{\prime}\right) \Omega^{\prime}-\frac{e^{-2 v_{3}}}{f}\left[\beta_{0}^{2} e^{-2\left(\alpha \phi+v_{1}+v_{2}\right)}+e^{-2 v_{2}} b^{2}+4 \sinh ^{2}\left(v_{1}-v_{2}\right)\right] \Omega=0 .
$$

Now suppose there were a nontrivial solution of this equation that satisfies the two boundary conditions $\Omega_{h}=0$ and $\Omega(\infty)=\Omega_{0}=0$. This would imply that $\Omega(r)$ must admit a maximal (or minimal) value somewhere if $\Omega>0$ (or $<0$, respectively). Having a maximal 
(minimal) value implies that at some radius, say $r=r_{e x}, \Omega^{\prime}\left(r_{e x}\right)=0$, and $\Omega^{\prime \prime}\left(r_{e x}\right)<0$ $(>0)$, so that it admits local Maximal (Minimal), and thus that

$$
\Omega\left(r_{e x}\right) \Omega^{\prime \prime}\left(r_{e x}\right)<0 .
$$

However this contradicts with (3.18), since

$$
\frac{e^{-2 v_{3}}}{f}\left[\beta_{0}^{2} e^{-2\left(\alpha \phi+v_{1}+v_{2}\right)}+e^{-2 v_{2}} b^{2}+4 \sinh ^{2}\left(v_{1}-v_{2}\right)\right]>0 .
$$

Therefore it is impossible to obtain a solution $\Omega$ which is not identically zero; the only allowed solution is $\Omega=0$ in all the radius.

Next, we consider the case $v_{1}=v_{2}$ on the horizon, for which the situation is a little bit complicated. In this case, the translational symmetry along $x^{1}$ direction is recovered on the horizon because the three dimensional spatial metric becomes flat space under our metric ansatz (3.6). Then, the theorem in section 2 cannot exclude the possibility that the horizon rotates along $x^{1}$ direction.

Let us investigate the equations for $\xi:=v_{1}-v_{2}$ and $b$,

$$
\begin{aligned}
f \xi^{\prime \prime}+\left\{f^{\prime}+f\left(v_{1}^{\prime}+\right.\right. & \left.\left.v_{2}^{\prime}+v_{3}^{\prime}\right)\right\} \xi^{\prime}-2\left(e^{-2 v_{3}}-\frac{\Omega^{2}}{f}\right) \sinh 2 \xi \\
- & \frac{e^{-2\left(v_{2}+v_{3}\right)}}{2}\left(1-e^{2 v_{3}} \frac{\Omega^{2}}{f}\right) b^{2}+\frac{1}{2} e^{-2 v_{1}} f b^{\prime 2}=0, \\
f b^{\prime \prime}+\left\{f^{\prime}+f\left(v_{3}^{\prime}+v_{2}^{\prime}-v_{1}^{\prime}\right)\right\} b^{\prime}-e^{2\left(v_{1}-v_{2}\right)}\left(e^{-2 v_{3}}-\frac{\Omega^{2}}{f}\right) b & =0 .
\end{aligned}
$$

As shown in [26], the near horizon behavior of $b$ is

$$
b \sim\left(r-r_{h}\right)^{\eta_{ \pm}}, \quad \eta_{ \pm}= \pm \frac{i \Omega_{h}}{\kappa},
$$

where $\kappa=f^{\prime}\left(r_{h}\right)$. Since both the solutions are singular at the horizon, we conclude that their coefficients must vanish in order to have a smooth horizon. This means that $b(r)=b^{\prime}(r)=0$ near $r \rightarrow r_{h}$. Since we solve the 2 nd order differential equation, this means that $b(r)=0$ in all the radius. Substituting $b=0$ into eq. (3.21), we obtain similar singular solutions near the horizon as

$$
\xi \sim\left(r-r_{h}\right)^{2 \eta_{ \pm}},
$$

where we used the fact that $\xi=0$ on the horizon. Thus, to have a smooth horizon, $\Omega_{h}=0$. Repeating the same argument on eq. (3.18), we obtain the same conclusion that $\Omega=0$ at all the radius outside the horizon.

Given $\Omega=0$, it is easy to see that the expectation value of the current on the dual field theory, $\left\langle j_{x^{1}}\right\rangle$ along $x^{1}$ direction must be zero. By eq. (3.16), we obtain $A_{x^{1}}^{\prime}=0$. Since $\left\langle j_{x^{1}}\right\rangle$ corresponds to the subleading term in the asymptotic behavior of $A_{x^{1}}$,

$$
A_{x^{1}} \simeq A_{1}+\frac{A_{2}}{r^{2}}
$$

$A_{x^{1}}^{\prime}=0$ means $A_{2}=0$. Thus, we obtain $\left\langle j_{x^{1}}\right\rangle=0$, implying that the charge current is zero along $x^{1}$ direction, as expected. 


\subsection{Bianchi $\mathrm{VI}_{0}$}

Next, in this subsection, we consider the Bianchi $\mathrm{VI}_{0}$ case. The corresponding three Killing vectors $\xi_{i}(i=1,2,3)$ are

$$
\xi_{1}=\partial_{x^{2}}, \quad \xi_{2}=\partial_{x^{3}}, \quad \xi_{3}=\partial_{x^{1}}+x^{2} \partial_{x^{2}}-x^{3} \partial_{x^{3}}
$$

and the invariant one-forms are

$$
\omega^{1}=e^{-x^{1}} d x^{2}, \quad \omega^{2}=e^{x^{1}} d x^{3}, \quad \omega^{3}=d x^{1} .
$$

Clearly there is no translational invariance along $x^{1}$. Then we obtain $\Omega_{h}=0$ at the horizon by the theorem in section 2 .

From the Maxwell equation (3.3),

$$
\begin{aligned}
e^{v_{1}+v_{2}+v_{3}+2 \alpha \phi}\left(A_{t}^{\prime}-\Omega A_{x^{1}}^{\prime}\right) & =\beta_{0}, \\
e^{v_{1}+v_{2}+v_{3}+2 \alpha \phi}\left\{\Omega\left(A_{t}^{\prime}-\Omega A_{x^{1}}^{\prime}\right)+f e^{-2 v_{3}} A_{x^{1}}^{\prime}\right\} & =C .
\end{aligned}
$$

Since $\Omega_{h}=0$, we obtain $C=0$. Thus, similar to the Bianchi $\mathrm{VII}_{0}$ case, we obtain a linear differential equation for $\Omega$,

$$
f \Omega^{\prime \prime}+f\left(v_{1}^{\prime}+v_{2}^{\prime}+3 v_{3}^{\prime}\right) \Omega^{\prime}-e^{-2 v_{3}}\left[4+e^{-2 v_{1}} b^{2}+\beta_{0}^{2} e^{-2\left(\alpha \phi+v_{1}+v_{2}\right)}\right] \Omega=0
$$

from the Einstein equation. Just like the Bianchi $\mathrm{VII}_{0}$ case, we have

$$
e^{-2 v_{3}}\left[4+e^{-2 v_{1}} b^{2}+\beta_{0}^{2} e^{-2\left(v_{1}+v_{2}\right)}\right]>0 .
$$

This implies that $\Omega$ cannot have a solution satisfying $\Omega\left(r_{h}\right)=\Omega(\infty)=0$, and thus, $\Omega=0$ in all the radius.

\subsection{Bianchi II}

In the case of the Bianchi II, the three Killing vectors are

$$
\xi_{1}=\partial_{x^{2}}, \quad \xi_{2}=\partial_{x^{3}}, \quad \xi_{3}=\partial_{x^{1}}+x^{3} \partial_{x^{3}}
$$

and the invariance one-forms are

$$
\omega^{1}=d x^{2}-x^{1} d x^{3}, \quad \omega^{2}=d x^{3}, \quad \omega^{3}=d x^{1} .
$$

Clearly there is no translational invariance along $x^{1}$. Then we obtain $\Omega_{h}=0$ at the horizon by the theorem in section 2 .

The Maxwell equation (3.3) gives the same form as the Bianchi $\mathrm{VII}_{0}$ case. Similarly, we obtain a linear differential equation for $\Omega$

$$
f \Omega^{\prime \prime}+f\left(v_{1}^{\prime}+v_{2}^{\prime}+3 v_{3}^{\prime}\right) \Omega^{\prime}-e^{-2 v_{3}}\left[e^{2\left(v_{1}-v_{2}\right)}+e^{-2 v_{2}} b^{2}+\beta_{0}^{2} e^{-2\left(\alpha \phi+v_{1}+v_{2}\right)}\right] \Omega=0 .
$$

This equation denies the solution where $\Omega$ has local maxima/minima so that the boundary conditions $\Omega\left(r_{h}\right)=\Omega(\infty)=0$ are satisfied. 
We have shown that $\Omega$ is identically zero outside the horizon in Bianchi II, $\mathrm{VI}_{0}$, and $\mathrm{VII}_{0}$ cases by using the assumption $\beta_{0} \neq 0$. Note that this assumption is not necessarily required in Bianchi II, $\mathrm{VI}_{0}$ cases because we can obtain $\Omega\left(r_{e x}\right) \Omega^{\prime \prime}\left(r_{e x}\right)<0$ regardless of $\beta_{0} \neq 0$. In Bianchi $\mathrm{VII}_{0}$ case, however, we need this assumption to prove $\Omega\left(r_{e x}\right) \Omega^{\prime \prime}\left(r_{e x}\right)<0$. So, it is interesting whether we can show that $\Omega$ is identically zero outside the horizon for the Bianchi $\mathrm{VII}_{0}$ case even when $\beta_{0}=0$, or the vacuum case. We leave this question open for future work.

\section{Symmetries broken in the bulk, restored on the horizon}

So far we have seen that it is difficult to have a gravitational solution on the Bianchi type $\mathrm{II}, \mathrm{VI}_{0}, \mathrm{VII}_{0}$, where $\Omega$ is nonzero along a direction on which there is no translational invariance. One possible exception is that the gauge potential $A$ in section 3 has a mass, just like the case in which a gauge field acquires a mass according to the superconducting state [26]. However in that case, the black brane horizon itself is actually not rotating and momentum is carried only by the matter fields outside. Therefore, it would be interesting to explore whether there exists a stationary black brane solution, where the horizon is rotating and the momentum flows along the direction with no translational symmetry, evading the symmetry theorem in section 2. As explained in section 3 , this is only possible if $v_{1}=v_{2}$ on the horizon in the Bianchi type $\mathrm{VII}_{0}$ because translational symmetry is recovered on $\partial_{x^{1}}$ direction on the horizon.

In this section, we will construct such a solution in the Einstein-Maxwell theory with an additional vector field $C$ that has a potential with a source term. The horizon is rotating along $x^{1}$ direction on which translational symmetry is broken outside the horizon. Such a solution is different from the rotating stationary solutions numerically found in $[37,38]$ in the sense that no dissipation occurs in our solution.

\subsection{Our model}

In the Bianchi $\mathrm{VII}_{0}$, the model we consider is

$$
S=\int d^{5} x \sqrt{-g}\left(R+\frac{12}{L^{2}}-\frac{1}{4} F^{2}-\frac{1}{4} W^{2}-V(C)\right)
$$

where $A$ and $C$ are one-form potentials,

$$
F=d A, \quad W=d C,
$$

and the potential term $V(C)$ for the one-form $C$ is given by

$$
\begin{aligned}
V(C) & =a_{0}\left(C-C_{0}\right)^{2}+a_{1}\left(C-C_{0}\right)^{4}, \\
C_{0} & \equiv c_{0} \omega^{1},
\end{aligned}
$$

where $\omega^{1}$ is the invariant one-form. Note that the gauge symmetry for $C$ is explicitly broken by their potential $V(C)$. We regard here, $a_{0}, a_{1}, c_{0}$ as parameters inducing the lattice effects. Especially $c_{0} \neq 0$ is crucial for the source term to be introduced. Note also that the gauge field $A$ corresponds to normal states in the dual field theory and accordingly does not have a mass term in (4.1). 
This Lagrangian does not have a translational invariant vacuum if these parameters are nonzero, especially $c_{0} \neq 0$. We regard that this is a sort of effective Lagrangian for the model where the lattice effects are introduced so that we do not have a translationally invariant vacuum. How translational invariant UV theory could induce such a source term by symmetry breaking is another question. In this section, putting aside such a question, we regard the action (4.1) as given and seek for a solution under the metric ansatz (3.6).

We assume that the flux forms

$$
C_{\mu} d x^{\mu}=c(r) \omega^{1}
$$

in such a way that at the horizon,

$$
\lim _{r \rightarrow r_{h}} c(r) \rightarrow 0 .
$$

It is rather easy to see that without the potential $V(C)$, the only allowed regular solution is $v_{1}=v_{2}$ in all the radius, implying that the lattice effects disappear. To see this explicitly, let us first consider the case where $a_{0}=a_{1}=0$. Then, the equations of motion for $\xi=v_{1}-v_{2}$ and $c$ are equivalent to eqs. (3.21) and (3.22) by replacing $b$ with $c$. So, following the argument in section 3 , we obtain $\xi=c=0$ in all the radius when $a_{0}=a_{1}=0$.

\subsection{Equations of motion}

We now consider generic $a_{0} \neq 0, a_{1} \neq 0$ case for the action (4.1). The Einstein equation is

$$
\begin{aligned}
R_{\mu \nu}= & \frac{1}{2} F_{\mu \alpha} F_{\nu}{ }^{\alpha}+\frac{1}{2} W_{\mu \alpha} W_{\nu}^{\alpha}+\left\{a_{0}+2 a_{1}\left(C-C_{0}\right)^{2}\right\}\left(C-C_{0}\right)_{\mu}\left(C-C_{0}\right)_{\nu} \\
& -\frac{g_{\mu \nu}}{12}\left[\frac{48}{L^{2}}+F^{2}+W^{2}+4 a_{1}\left(C-C_{0}\right)^{4}\right] .
\end{aligned}
$$

Using the metric ansatz (3.6), it is straightforward to obtain the equation of motion for $\xi=v_{1}-v_{2}$ as

$$
\begin{aligned}
f \xi^{\prime \prime}+\left\{f^{\prime}+f\left(v_{1}^{\prime}+v_{2}^{\prime}+v_{3}^{\prime}\right)\right\} \xi^{\prime}-2 & \left(e^{-2 v_{3}}-f^{-1} \Omega^{2}\right) \sinh 2 \xi \\
-\frac{e^{-2\left(v_{2}+v_{3}\right)}}{2} & \left(1-e^{2 v_{3}} f^{-1} \Omega^{2}\right) c^{2}+\frac{1}{2} e^{-2 v_{1}} f c^{\prime 2} \\
& =-a_{0} e^{-2 v_{1}}\left(c-c_{0}\right)^{2}-2 a_{1} e^{-4 v_{1}}\left(c-c_{0}\right)^{4} .
\end{aligned}
$$

The equations of motion for $\Omega(r), v_{1}, v_{3}$ are respectively,

$$
\begin{aligned}
\Omega^{\prime \prime}+ & \left(v_{1}^{\prime}+v_{2}^{\prime}+3 v_{3}^{\prime}\right) \Omega^{\prime}-4 e^{-2 v_{3}} f^{-1} \sinh ^{2}\left(v_{1}-v_{2}\right) \Omega \\
& +e^{-2 v_{3}} A_{x}^{\prime}\left(A_{t}^{\prime}-\Omega A_{x}^{\prime}\right)-\frac{e^{-2\left(v_{2}+v_{3}\right)}}{f} c^{2} \Omega=0 \\
f v_{1}^{\prime \prime}+ & \left\{f^{\prime}+f\left(v_{1}^{\prime}+v_{2}^{\prime}+v_{3}^{\prime}\right)\right\} v_{1}^{\prime}+\frac{1}{6} A_{t}^{\prime 2}+\frac{1}{6}\left(\Omega^{2}-f e^{-2 v_{3}}\right) A_{x}^{\prime 2}-\frac{1}{3} \Omega A_{x}^{\prime} A_{t}^{\prime} \\
& +\sinh \left\{2\left(v_{1}-v_{2}\right)\right\} f^{-1} \Omega^{2}-e^{-2 v_{3}} \sinh \left\{2\left(v_{1}-v_{2}\right)\right\}-\frac{4}{L^{2}}+a_{0} e^{-2 v_{1}}\left(c-c_{0}\right)^{2} \\
& +\frac{5}{3} a_{1} e^{-4 v_{1}}\left(c-c_{0}\right)^{4}+\frac{1}{3} e^{-2 v_{1}} f c^{\prime 2}-\frac{e^{-2\left(v_{2}+v_{3}\right)} c^{2}}{6}\left(1-e^{2 v_{3}} f^{-1} \Omega^{2}\right)=0
\end{aligned}
$$




$$
\begin{aligned}
f v_{3}^{\prime \prime}+ & \left\{f^{\prime}+f\left(v_{1}^{\prime}+v_{2}^{\prime}+v_{3}^{\prime}\right)\right\} v_{3}^{\prime}+\frac{1}{6}\left(A_{t}^{\prime}-\Omega A_{x}^{\prime}\right)^{2}+\frac{1}{3} f e^{-2 v_{3}} A_{x}^{\prime 2} \\
& +2 e^{-2 v_{3}} \sinh ^{2}\left(v_{1}-v_{2}\right)+\frac{e^{2 v_{3}}}{2} \Omega^{\prime 2}-\frac{4}{L^{2}}-\frac{a_{1}}{3} e^{-4 v_{1}}\left(c-c_{0}\right)^{4} \\
& -\frac{1}{6} f e^{-2 v_{1}} c^{\prime 2}+\frac{c^{2}}{6} e^{-2\left(v_{2}+v_{3}\right)}\left(2+e^{2 v_{3}} f^{-1} \Omega^{2}\right)=0 .
\end{aligned}
$$

From the constraint equation, we have for $f$ as

$$
\begin{aligned}
& \frac{1}{2} e^{2 v_{3}} \Omega^{\prime 2}-\frac{1}{2} f e^{-2 v_{3}} A_{x}^{\prime 2}+\frac{1}{2}\left(A_{t}^{\prime}-\Omega A_{x}^{\prime}\right)^{2}-\frac{12}{L^{2}}+2 \sinh ^{2}\left(v_{1}-v_{2}\right)\left(e^{-2 v_{3}}-f^{-1} \Omega^{2}\right) \\
& +f^{\prime}\left(v_{1}^{\prime}+v_{2}^{\prime}+v_{3}^{\prime}\right)+2 f\left(v_{1}^{\prime} v_{2}^{\prime}+v_{2}^{\prime} v_{3}^{\prime}+v_{3}^{\prime} v_{1}^{\prime}\right)+a_{0} e^{-2 v_{1}}\left(c-c_{0}\right)^{2} \\
& +a_{1} e^{-4 v_{1}}\left(c-c_{0}\right)^{4}-\frac{f}{2} e^{-2 v_{1}} c^{\prime 2}+\frac{e^{-2\left(v_{2}+v_{3}\right)}}{2}\left(1-e^{2 v_{3}} f^{-1} \Omega^{2}\right) c^{2}=0 .
\end{aligned}
$$

The equations of motion for the potential $A$ give

$$
\begin{aligned}
e^{v_{1}+v_{2}+v_{3}}\left(A_{t}^{\prime}-\Omega A_{x}^{\prime}\right) & =\beta_{0}, \\
\beta_{0} \Omega+e^{v_{1}+v_{2}-v_{3}} f A_{x}^{\prime} & =\beta_{1},
\end{aligned}
$$

and for $C$,

$$
\begin{aligned}
f c^{\prime \prime}+\left\{f^{\prime}+f\left(v_{3}^{\prime}+v_{2}^{\prime}-v_{1}^{\prime}\right)\right\} c^{\prime}-e^{2\left(v_{1}-v_{2}\right)}\left(e^{-2 v_{3}}-f^{-1} \Omega^{2}\right) c \\
=2 a_{0}\left(c-c_{0}\right)+4 a_{1} e^{-2 v_{1}}\left(c-c_{0}\right)^{3} .
\end{aligned}
$$

Now, we have additional terms due to $a_{0}$ and $a_{1}$, and eq. (4.15) is a non-linear differential equation with respect to $c$. Note that $c$ must vanish on the horizon to have a regular solution for $c$, otherwise the l.h.s. of eq. (4.15) would diverge at the horizon, due to the term $f^{-1} \Omega^{2} c$ when $\Omega_{h} \neq 0$. This implies that the r.h.s. of eq. (4.8) becomes non-vanishing on the horizon. By the theorem in section 2, we must set $\xi=0$ on the horizon. Because of these, we can now expand $c, v_{i}$, and $\Omega$ as

$$
\begin{aligned}
c(r) & =c_{1}\left(r-r_{h}\right)+O\left(r-r_{h}\right)^{2}, \\
v_{1} & =v_{2}+O\left(r-r_{h}\right), \\
\Omega(r) & =\Omega_{h}+O\left(r-r_{h}\right), \\
c_{1} & =-\frac{2 \kappa c_{0}}{\kappa^{2}+\Omega_{h}^{2}}\left(a_{0}+2 a_{1} e^{-2 v_{1}} c_{0}^{2}\right)
\end{aligned}
$$

to have a regular solution, here the coefficient $c_{1}$ is determined by eq. (4.15). On the other hand, at spatial infinity $r \rightarrow \infty, c(r)$ behaves as

$$
c(r) \simeq \alpha_{+} r^{n_{+}}+\alpha_{-} r^{n_{-}}+\left(1-\frac{e^{2 \xi(\infty)}}{2 C_{3} a_{0}} r^{-2}\right) c_{0}, \quad n_{ \pm}=-1 \mp \sqrt{1+2 a_{0} L^{2}} .
$$

According to the AdS/CFT dictionary, the non-normalizable mode $r^{n_{-}}$and the normalizable mode $r^{n_{+}}$correspond to the source term and the expectation value on the field theory side, respectively. So, we impose a boundary condition,

$$
\alpha_{-}=0 .
$$


In addition, we also impose that

$$
\Omega(r=\infty)=0,
$$

since we are interested in the boundary theory on a static spacetime.

There still remains a gauge freedom for the choice of the coordinates $t, r, x^{2}$, and $x^{3}$ under the boundary conditions (4.18) and (4.19), due to the scaling freedom

$$
\begin{aligned}
& r \rightarrow \alpha_{1} r, \quad t \rightarrow \frac{t}{\alpha_{1}}, \quad x^{2} \rightarrow \alpha_{2} x^{2}, \quad x^{3} \rightarrow \alpha_{2} x^{3}, \\
& f \rightarrow \alpha_{1}^{2} f, \quad e^{v_{1}} \rightarrow \frac{e^{v_{1}}}{\alpha_{2}}, \quad e^{v_{2}} \rightarrow \frac{e^{v_{2}}}{\alpha_{2}},
\end{aligned}
$$

which preserves the metric ansatz (3.6) and the asymptotic AdS boundary condition (3.7) with $\Omega_{0}=0$. Thus, to perform the numerics and determine the temperature uniquely, we shall fix the asymptotic AdS metric as

$$
d s^{2} \simeq-\frac{r^{2}}{L^{2}} d t^{2}+\frac{L^{2}}{r^{2}} d r^{2}+r^{2}\left(d x^{1}+\Omega d t\right)^{2}+r^{2} e^{\xi}\left(\omega^{1}\right)^{2}+r^{2} e^{-\xi}\left(\omega^{2}\right)^{2} .
$$

\subsection{Numerical solution}

Under the boundary conditions (4.18) and (4.19), we numerically construct a rotating black brane solution in asymptotically AdS spacetime. By imposing regularity on the horizon, one can derive the following boundary conditions;

$$
\begin{aligned}
& \xi^{\prime}\left(r_{h}\right)=-\frac{\kappa b_{0}^{2}}{\kappa^{2}+4 \Omega_{h}^{2}}\left(a_{0}+2 a_{1} e^{-2 v_{1}\left(r_{h}\right)} b_{0}^{2}\right) e^{-2 v_{1}\left(r_{h}\right)}, \\
& \kappa v_{1}^{\prime}\left(r_{h}\right)+ \frac{\beta_{0}^{2}}{6} e^{-2\left(v_{1}\left(r_{h}\right)+v_{2}\left(r_{h}\right)+v_{3}\left(r_{h}\right)\right)}+\frac{2 \xi^{\prime}\left(r_{h}\right) \Omega_{h}^{2}}{\kappa}-\frac{4}{L^{2}} \\
&+a_{0} e^{-2 v_{1}\left(r_{h}\right)} b_{0}^{2}+\frac{5}{3} a_{1} e^{-4 v_{1}\left(r_{h}\right)} b_{0}^{4}=0, \\
& \kappa v_{3}^{\prime}\left(r_{h}\right)+ \frac{\beta_{0}^{2}}{6} e^{-2\left(v_{1}\left(r_{h}\right)+v_{2}\left(r_{h}\right)+v_{3}\left(r_{h}\right)\right)} \\
&+\frac{e^{2 v_{3}\left(r_{h}\right)}}{2} \Omega^{\prime 2}\left(r_{h}\right)-\frac{4}{L^{2}}-\frac{a_{1}}{3} e^{-4 v_{1}\left(r_{h}\right)} b_{0}^{4}=0, \\
& \beta_{1}=\beta_{0} \Omega_{h},
\end{aligned}
$$

where the last equation is derived from eq. (4.14). Thus, the free parameters on the horizon are

$$
r_{h}, v_{1}\left(r_{h}\right), v_{3}\left(r_{h}\right), \Omega_{h}, \Omega^{\prime}\left(r_{h}\right), \beta_{0}, \kappa
$$

Note that the solution for $c$ is uniquely determined by the regularity condition at the horizon (4.16) for the given parameters (4.27), and the asymptotic behavior does not generically match on to the condition (4.18). So, to match on to the UV boundary condition (4.18), we need to choose a specific value $a_{1}$ for each parameter choice (4.27). In addition, to satisfy the UV boundary condition (4.19), we will find a specific parameter $\Omega^{\prime}\left(r_{h}\right)$ for the 


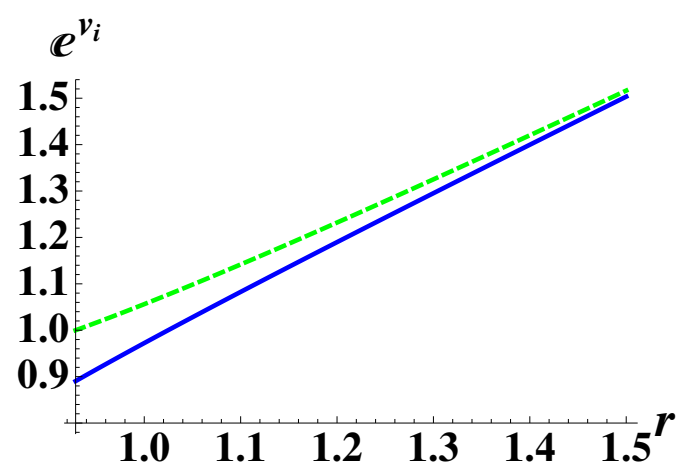

Figure 1. $e^{v_{1}}$ (solid) and $e^{v_{3}}$ (dashed) are shown for the parameter choice (I).

\section{$\{\Omega, \xi\}$}

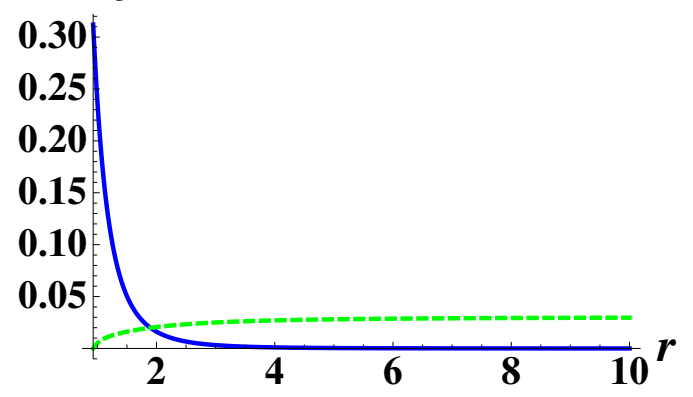

Figure 3. $\Gamma=\Omega$ (solid) and $\Gamma=\xi$ (dashed) are shown for the parameter choice (I).

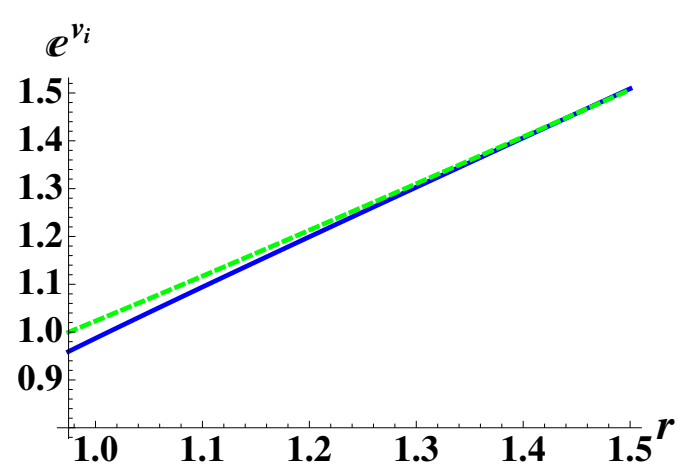

Figure 2. $e^{v_{1}}$ (solid) and $e^{v_{3}}$ (dashed) are shown for the parameter choice (II).

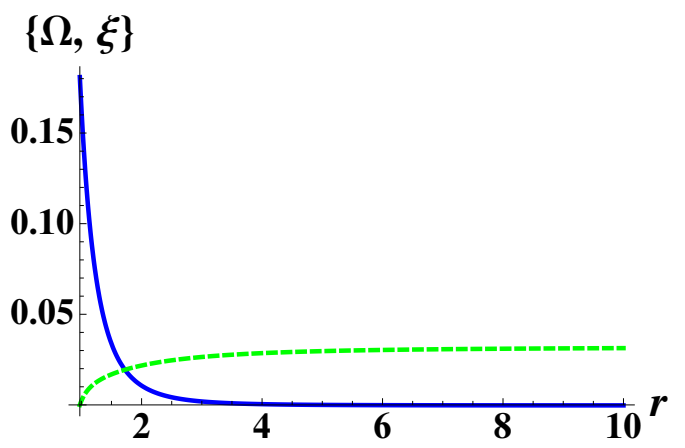

Figure 4. $\Gamma=\Omega$ (solid) and $\Gamma=\xi$ (dashed) are shown for the parameter choice (II).

given values $v_{1}\left(r_{h}\right), v_{3}\left(r_{h}\right), \Omega_{h}, \beta_{0}, \kappa$ by a shooting method. ${ }^{3}$ This implies that we need to choose a theory $a_{0}, a_{1}$, and $c_{0}$ for a given temperature $T=\frac{\kappa}{4 \pi}$, angular velocity $\Omega\left(r_{h}\right)$, and the charge density $\beta_{0}$.

Let us pay attention to the case $a_{0}>0$, otherwise, the null energy condition would be violated asymptotically, as seen below. Then, if the condition (4.18) is satisfied, $c$ asymptotically approaches $c_{0}$. We find two solutions by a shooting method and the parameter choices are

$$
\begin{aligned}
& \text { (I) }: \quad L=\sqrt{2}, \quad v_{1}\left(r_{h}\right)=-0.1161, \quad v_{3}\left(r_{h}\right)=0, \\
& a_{0}=0.1, \quad a_{1}=-10.82 \\
& \Omega\left(r_{h}\right)=0.3120, \quad \Omega^{\prime}\left(r_{h}\right)=-1.213, \quad \beta_{0}=0.4861, \quad \kappa=1.560, \\
& \text { (II) : } \quad L=\sqrt{2}, \quad v_{1}\left(r_{h}\right)=-0.04137, \quad v_{3}\left(r_{h}\right)=0, \\
& a_{0}=0.1, \quad a_{1}=-11.17, \\
& \Omega\left(r_{h}\right)=0.1808, \quad \Omega^{\prime}\left(r_{h}\right)=-0.7043, \quad \beta_{0}=0.5644, \quad \kappa=1.811,
\end{aligned}
$$

as shown in figures $1-8$.

\footnotetext{
${ }^{3}$ Here, $r_{h}$ has gauge degrees of freedom, and we fix it by imposing $f$ to approach $r^{2} / L^{2}+O(1)$ asymptotically.
} 


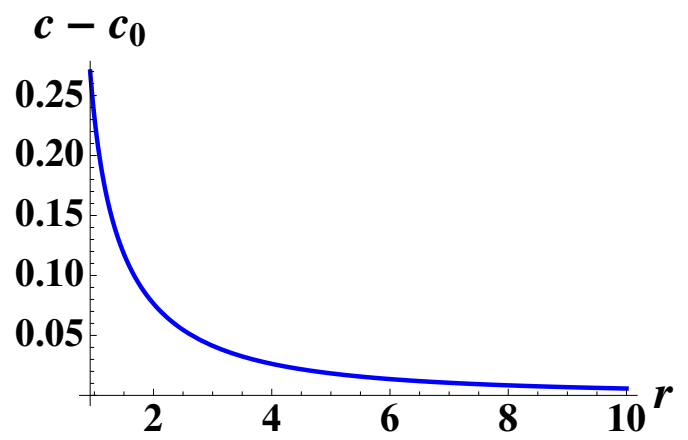

Figure 5. $c-c_{0}$ is shown for the parameter choice (I).

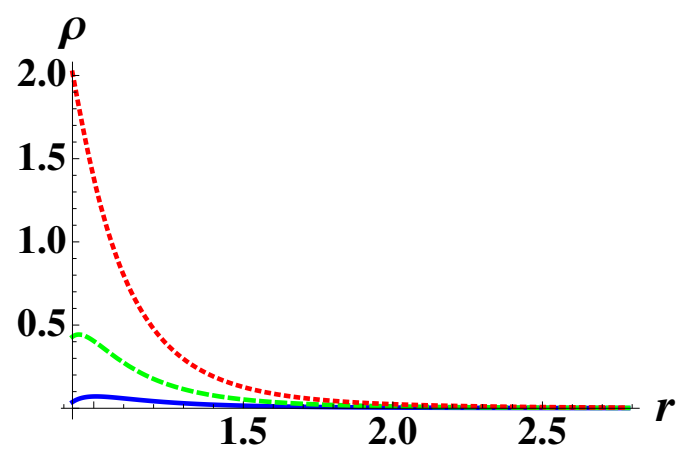

Figure 7. $\rho=R_{\mu \nu} K^{\mu} K^{\nu}$ for $\gamma=1$ (solid), $\gamma=2$ (dashed), and $\gamma=3$ (dotted) are shown for the parameter choice (I).

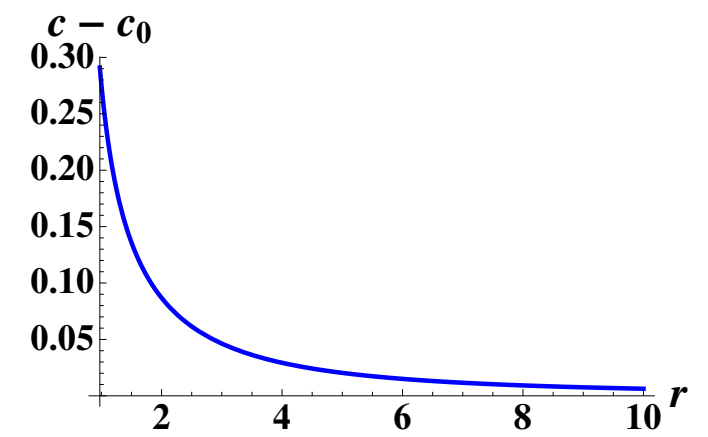

Figure 6. $c-c_{0}$ is shown for the parameter choice (II).

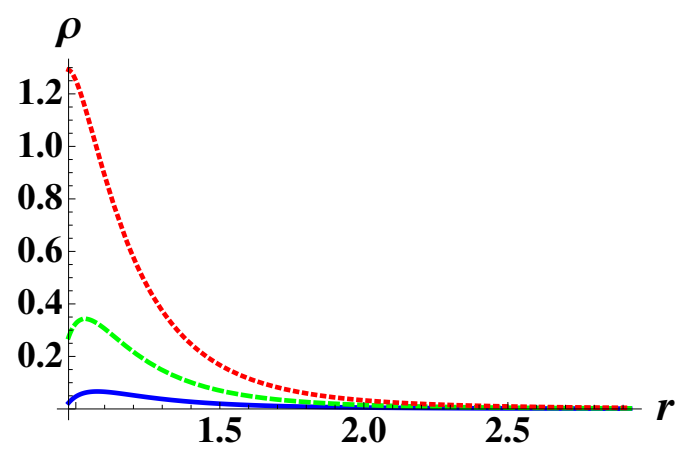

Figure 8. $\rho=R_{\mu \nu} K^{\mu} K^{\nu}$ for $\gamma=1$ (solid), $\gamma=2$ (dashed), and $\gamma=3$ (dotted) are shown for the parameter choice (II).

As shown in figure $1-6$, all $v_{i}$ logarithmically increases as $v_{i} \sim \ln r$, while $\Omega$ and $c-c_{0}$ approach zero. This implies that the spacetime asymptotically becomes AdS spacetime in the sense mentioned in section 3. Since we are interested in the solution satisfying the null energy condition, i. e., $R_{\mu \nu} K^{\mu} K^{\nu} \geq 0$ for any null vector $K$, it is worth investigating whether it is satisfied in our solution. Contracting eq. (4.7) with a null vector $K$, we find

$$
\begin{aligned}
\rho:=R_{\mu \nu} K^{\mu} K^{\nu}=\frac{1}{2}( & \left.F_{\mu \alpha} F_{\nu}{ }^{\alpha}+W_{\mu \alpha} W_{\nu}^{\alpha}\right) K^{\mu} K^{\nu} \\
& +\left\{a_{0}+2 a_{1}\left(C-C_{0}\right)^{2}\right\}\left\{\left(C-C_{0}\right)_{\mu} K^{\mu}\right\}^{2} .
\end{aligned}
$$

As the first two terms in the r.h.s. are kinetic terms, they would be positive. So, only the term with a negative coefficient $a_{1}$ would be negative. This implies that the null energy condition might be violated when $g\left(C-C_{0}, K\right)$ is large. To investigate the sign of eq. (4.30), we prepare the following pseudo-orthonormal vectors,

$$
\begin{aligned}
l_{+} & =\partial_{t}-\Omega \partial_{x^{1}}+f \partial_{r}, \\
l_{-} & =\frac{1}{2}\left(\frac{1}{f} \partial_{t}-\frac{\Omega}{f} \partial_{x^{1}}-\partial_{r}\right), \\
E_{3} & =e^{-v_{3}} \partial_{x^{1}}
\end{aligned}
$$




$$
\begin{aligned}
& E_{1}=e^{-v_{1}}\left(\cos x^{1} \partial_{x^{2}}+\sin x^{1} \partial_{x^{3}}\right), \\
& E_{2}=e^{-v_{2}}\left(\sin x^{1} \partial_{x^{2}}-\cos x^{1} \partial_{x^{3}}\right)
\end{aligned}
$$

satisfying the relation

$$
\begin{aligned}
& g\left(l_{ \pm}, l_{ \pm}\right)=g\left(l_{ \pm}, E_{i}\right)=g\left(E_{i}, E_{j}\right)=0(i \neq j), \\
& g\left(l_{-}, l_{+}\right)=-1, \quad g\left(E_{i}, E_{i}\right)=1 .
\end{aligned}
$$

Note that $l_{+}$and $l_{-}$represent outward and inward pointing null vectors, respectively. Thus, $l_{+}$becomes a tangent vector of the null geodesic generator on the horizon.

As $E_{2}$ and $E_{3}$ are orthogonal to $C-C_{0}$ vector, we shall consider a null vector $K$ as

$$
K=l_{+}+\zeta l_{-}+\gamma E_{1}
$$

By requiring $g(K, K)=0$, we find $\zeta=\gamma^{2} / 2$. In figure 4, we plot $\rho:=R_{\mu \nu} K^{\mu} K^{\nu}$ for several parameters $\gamma$ and find that $\rho$ is always positive for each parameter choice (I) and (II). This indicates that the null energy condition is satisfied in our numerical solution.

The Kretschmann scalar curvature invariant $K=R_{\mu \nu \alpha \beta} R^{\mu \nu \alpha \beta}$ can be expanded near the horizon $r=r_{h}$ as a series in $\left(r-r_{h}\right)$. Under the ansatz (4.16), we find that $K$ can be expanded as $K=\alpha_{0}+\alpha_{1}\left(r-r_{h}\right)+\cdots<\infty$, where $\alpha_{i}(i=0,1)$ is a finite coefficient. In this sense, the numerical solution is regular near the horizon. As mentioned in section 1, this indicates that the black brane solution is not analytic but regular on the horizon since the horizon can be compactified, due to the periodicity of the lattice structure. Thus, the solution would be the first example of a stationary black hole solution with a compact rotating horizon which evades the rigidity theorem by violating one of the crucial conditions, analyticity.

\section{Summary and discussion}

In this paper, we have studied in what circumstances a Bianchi black brane can have momentum along a direction with translational invariance being broken. First, after having given the theorem concerning the decomposition of a Killing vector on the horizon, we have shown that in the Einstein-Maxwell-dilation theory, stationary Bianchi black branes of the type II, $\mathrm{VI}_{0}$, and $\mathrm{VII}_{0}$ cannot convey momentum along the direction of no translational invariance. For these cases, we have shown under our metric ansatz (2.2) that the timeasymmetric part of the metric, $\Omega(r)$, must be identically zero in the entire region outside the horizon, and thus the geometry must be static. Two main obstacles for constructing such solutions are the rigidity theorem and our choice of boundary conditions: the former prevents the horizon from rotating and the latter, reflecting the requirement for the absence of non-normalizable mode, gives the tight restriction so that $\Omega=0$ both at the horizon and infinity.

Next we have considered Bianchi $\mathrm{VII}_{0}$ black branes in the Einstein-Maxwell theory with an additional vector field having a source term. We have numerically constructed 
a solution whose horizon admits a translational invariance and thus conveys momentum with non-vanishing $\Omega$ on the horizon, which can be viewed as "rotation" if the horizon cross-sections are compactified. Interestingly, the translational invariance is broken just outside the horizon. Therefore our solution indicates the existence of a black brane solution which is regular but non-analytic at the horizon, thereby evading the black hole rigidity theorem. Our construction largely relies upon numerical calculation. It is hoped to justify by using some analytic methods, the existence of such a Bianchi black brane solution with a translational invariance being broken in the bulk, but restored only on the horizon. It is interesting to consider a dual field theory interpretation of this solution, and also questions like what is the field theory dual of analyticity of radial direction in bulk.

\section{Acknowledgments}

The work of NI was supported by RIKEN iTHES Project. This work was also supported in part by JSPS KAKENHI Grant Number 25800143 (NI), 22540299 (AI), 23740200 (KM), and 26400280 (NI, AI, KM).

\section{A Bianchi classification}

The Bianchi classification of 3-dimensional homogeneous spaces is in essence a classification of connected, simply connected 3-dimensional Lie-groups, which are classified into 9 types by the structure constants $C^{K}{ }_{I J}(I, J, K=1,2,3)$ of the corresponding Lie-algebras

$$
\left[\xi_{I}, \xi_{J}\right]=C^{K}{ }_{I J} \xi_{K}
$$

with $\left\{\xi_{I}\right\}$ being the generators of the Lie-algebras. Then, one can choose the invariant basis vectors $\left\{X_{I}\right\}$ so that

$$
\left[X_{I}, X_{J}\right]=-C^{K}{ }_{I J} X_{K},
$$

which can also be expressed in terms of the invariant dual basis one-form $\left\{\omega^{I}\right\}$ as

$$
d \omega^{I}=\frac{1}{2} C^{I} J K \omega^{J} \wedge \omega^{K} .
$$

In this appendix, we provide the structure constants, the generators, the invariant basis, and the invariant one-forms for the 3 types directly relevant to our black brane solutions, among 9-types of the Bianchi class.

- Type II: $C^{1}{ }_{23}=-C^{1}{ }_{32}=1$ and the rest $C^{I}{ }_{J K}=0$

$$
\begin{array}{lll}
\xi_{1}=\partial_{x^{2}} & X_{1}=\partial_{x^{2}} & \omega^{1}=d x^{2}-x^{1} d x^{3} \\
\xi_{2}=\partial_{x^{3}} & X_{2}=x^{1} \partial_{x^{2}}+\partial_{x^{3}} & \omega^{2}=d x^{3} \\
\xi_{3}=\partial_{x^{1}}+x^{3} \partial_{x^{2}} & X_{3}=\partial_{x^{1}} & \omega^{3}=d x^{1}
\end{array}
$$


- Type $V I_{0}: C^{1}{ }_{13}=-C^{1}{ }_{31}=1, C^{2}{ }_{23}=-C^{2}{ }_{32}=-1$ and the rest $C^{I}{ }_{J K}=0$

$$
\begin{array}{lll}
\xi_{1}=\partial_{x^{2}} & X_{1}=e^{x^{1}} \partial_{x^{2}} & \omega^{1}=e^{-x^{1}} d x^{2} \\
\xi_{2}=\partial_{x^{3}} & X_{2}=e^{-x^{1}} \partial_{x^{3}} & \omega^{2}=e^{x^{1}} d x^{3} \\
\xi_{3}=\partial_{x^{1}}+x^{2} \partial_{x^{2}}-x^{3} \partial_{x^{3}} & X_{3}=\partial_{x^{1}} & \omega^{3}=d x^{1}
\end{array}
$$

- Type VII $I_{0}: C^{1}{ }_{23}=-C^{1}{ }_{32}=-1, C^{2}{ }_{13}=-C^{2}{ }_{31}=1$ and the rest $C^{I}{ }_{J K}=0$.

$$
\begin{array}{ll}
\xi_{1}=\partial_{x^{2}} & X_{1}=\cos \left(x^{1}\right) \partial_{x^{2}}+\sin \left(x^{1}\right) \partial_{x^{3}} \\
\xi_{2}=\partial_{x^{3}} & X_{2}=-\sin \left(x^{1}\right) \partial_{x^{2}}+\cos \left(x^{1}\right) \partial_{x^{3}} \\
\xi_{3}=\partial_{x^{1}}-x^{3} \partial_{x^{2}}+x^{2} \partial_{x^{3}} & X_{3}=\partial_{x^{1}}
\end{array}
$$

and

$$
\begin{aligned}
\omega^{1} & =\cos \left(x^{1}\right) d x^{2}+\sin \left(x^{1}\right) d x^{3}, \\
\omega^{2} & =-\sin \left(x^{1}\right) d x^{2}+\cos \left(x^{1}\right) d x^{3}, \quad \omega^{3}=d x^{1} .
\end{aligned}
$$

Open Access. This article is distributed under the terms of the Creative Commons Attribution License (CC-BY 4.0), which permits any use, distribution and reproduction in any medium, provided the original author(s) and source are credited.

\section{References}

[1] S.S. Gubser, Breaking an abelian gauge symmetry near a black hole horizon, Phys. Rev. D 78 (2008) 065034 [arXiv:0801.2977] [INSPIRE].

[2] S.A. Hartnoll, C.P. Herzog and G.T. Horowitz, Building a holographic superconductor, Phys. Rev. Lett. 101 (2008) 031601 [arXiv:0803.3295] [INSPIRE].

[3] S.A. Hartnoll, C.P. Herzog and G.T. Horowitz, Holographic superconductors, JHEP 12 (2008) 015 [arXiv:0810.1563] [INSPIRE].

[4] C.P. Herzog, Lectures on holographic superfluidity and superconductivity, J. Phys. A 42 (2009) 343001 [arXiv:0904.1975] [InSPIRE].

[5] G.T. Horowitz, Introduction to holographic superconductors, Lect. Notes Phys. 828 (2011) 313 [arXiv:1002.1722] [INSPIRE].

[6] G.T. Horowitz and M.M. Roberts, Zero temperature limit of holographic superconductors, JHEP 11 (2009) 015 [arXiv:0908.3677] [INSPIRE].

[7] S. Kachru, X. Liu and M. Mulligan, Gravity duals of Lifshitz-like fixed points, Phys. Rev. D 78 (2008) 106005 [arXiv:0808.1725] [INSPIRE].

[8] M. Taylor, Non-relativistic holography, arXiv:0812.0530 [INSPIRE].

[9] K. Goldstein, S. Kachru, S. Prakash and S.P. Trivedi, Holography of charged dilaton black holes, JHEP 08 (2010) 078 [arXiv:0911.3586] [INSPIRE].

[10] K. Goldstein et al., Holography of dyonic dilaton black branes, JHEP 10 (2010) 027 [arXiv: 1007.2490] [INSPIRE]. 
[11] C. Charmousis, B. Gouteraux, B.S. Kim, E. Kiritsis and R. Meyer, Effective holographic theories for low-temperature condensed matter systems, JHEP 11 (2010) 151 [arXiv: 1005.4690] [INSPIRE].

[12] B. Gouteraux and E. Kiritsis, Generalized holographic quantum criticality at finite density, JHEP 12 (2011) 036 [arXiv:1107.2116] [INSPIRE].

[13] N. Iizuka, N. Kundu, P. Narayan and S.P. Trivedi, Holographic Fermi and non-Fermi liquids with transitions in dilaton gravity, JHEP 01 (2012) 094 [arXiv:1105.1162] [INSPIRE].

[14] N. Ogawa, T. Takayanagi and T. Ugajin, Holographic Fermi surfaces and entanglement entropy, JHEP 01 (2012) 125 [arXiv:1111.1023] [INSPIRE].

[15] L. Huijse, S. Sachdev and B. Swingle, Hidden Fermi surfaces in compressible states of gauge-gravity duality, Phys. Rev. B 85 (2012) 035121 [arXiv:1112.0573] [INSPIRE].

[16] E. Shaghoulian, Holographic entanglement entropy and Fermi surfaces, JHEP 05 (2012) 065 [arXiv: 1112.2702] [INSPIRE].

[17] X. Dong, S. Harrison, S. Kachru, G. Torroba and H. Wang, Aspects of holography for theories with hyperscaling violation, JHEP 06 (2012) 041 [arXiv:1201.1905] [INSPIRE].

[18] E. Perlmutter, Domain wall holography for finite temperature scaling solutions, JHEP 02 (2011) 013 [arXiv: 1006.2124] [INSPIRE].

[19] E. Perlmutter, Hyperscaling violation from supergravity, JHEP 06 (2012) 165 [arXiv:1205.0242] [INSPIRE].

[20] K. Narayan, On Lifshitz scaling and hyperscaling violation in string theory, Phys. Rev. D 85 (2012) 106006 [arXiv: 1202.5935] [INSPIRE].

[21] M. Edalati, J.F. Pedraza and W. Tangarife Garcia, Quantum fluctuations in holographic theories with hyperscaling violation, Phys. Rev. D 87 (2013) 046001 [arXiv:1210.6993] [INSPIRE].

[22] P. Bueno, W. Chemissany and C.S. Shahbazi, On hvLif-like solutions in gauged supergravity, Eur. Phys. J. C 74 (2014) 2684 [arXiv:1212.4826] [INSPIRE].

[23] N. Iizuka et al., Bianchi attractors: a classification of extremal black brane geometries, JHEP 07 (2012) 193 [arXiv: 1201.4861] [INSPIRE].

[24] N. Iizuka et al., Extremal horizons with reduced symmetry: hyperscaling violation, stripes and a classification for the homogeneous case, JHEP 03 (2013) 126 [arXiv:1212.1948] [INSPIRE].

[25] A. Donos and S.A. Hartnoll, Interaction-driven localization in holography, Nature Phys. 9 (2013) 649 [arXiv:1212.2998] [InSPIRE].

[26] N. Iizuka, A. Ishibashi and K. Maeda, Persistent superconductor currents in holographic lattices, arXiv:1312.6124 [INSPIRE].

[27] G.T. Horowitz and J.E. Santos, General relativity and the cuprates, arXiv:1302.6586 [INSPIRE].

[28] N. Iizuka and K. Maeda, Towards the lattice effects on the holographic superconductor, JHEP 11 (2012) 117 [arXiv:1207.2943] [INSPIRE].

[29] S.W. Hawking and G.F.R. Ellis, The large scale structure of space-time, Cambridge University Press, Cambridge U.K. (1973). 
[30] S. Hollands, A. Ishibashi and R.M. Wald, A higher dimensional stationary rotating black hole must be axisymmetric, Commun. Math. Phys. 271 (2007) 699 [gr-qc/0605106] [INSPIRE].

[31] V. Moncrief and J. Isenberg, Symmetries of higher dimensional black holes, Class. Quant. Grav. 25 (2008) 195015 [arXiv:0805.1451] [INSPIRE].

[32] P. Bizon and A. Rostworowski, On weakly turbulent instability of Anti-de Sitter space, Phys. Rev. Lett. 107 (2011) 031102 [arXiv:1104.3702] [INSPIRE].

[33] M. Maliborski and A. Rostworowski, Time-periodic solutions in an Einstein AdS-massless-scalar-field system, Phys. Rev. Lett. 111 (2013) 051102 [arXiv:1303.3186] [INSPIRE].

[34] A. Buchel, L. Lehner and S.L. Liebling, Scalar collapse in AdS, Phys. Rev. D 86 (2012) 123011 [arXiv:1210.0890] [InSPIRE].

[35] O.J.C. Dias, G.T. Horowitz, D. Marolf and J.E. Santos, On the Nonlinear Stability of Asymptotically Anti-de Sitter Solutions, Class. Quant. Grav. 29 (2012) 235019 [arXiv: 1208.5772] [INSPIRE].

[36] O.J.C. Dias, G.T. Horowitz and J.E. Santos, Black holes with only one Killing field, JHEP 07 (2011) 115 [arXiv: 1105.4167] [INSPIRE].

[37] P. Figueras and T. Wiseman, Stationary holographic plasma quenches and numerical methods for non-Killing horizons, Phys. Rev. Lett. 110 (2013) 171602 [arXiv: 1212.4498] [INSPIRE].

[38] S. Fischetti, D. Marolf and J.E. Santos, AdS flowing black funnels: Stationary AdS black holes with non-Killing horizons and heat transport in the dual CFT,

Class. Quant. Grav. 30 (2013) 075001 [arXiv:1212.4820] [INSPIRE].

[39] S. Sachdev and M. Mueller, Quantum criticality and black holes, J. Phys. Cond. Matt. 21 (2009) 164216 [arXiv:0810.3005]. 